\title{
Analytical stripe phase solution for the Hubbard model.
}

\author{
S.I. Matveenko \\ Landau Institute for Theoretical Physics, Kosygina Str. 2, \\ 117940, Moscow, Russia \\ and \\ S.I. Mukhin \\ Theoretical Physics Dept., \\ Moscow Institute for Steel and Alloys, Leninskii pr. 4, \\ 117936 Moscow, Russia
}

(October 11, 2018)

\begin{abstract}
The self-consistent solution for the spin-charge solitonic superstructure in quasi-one-dimensional electron system is obtained in the framework of the Hubbard model as a function of a hole doping. Effects of interchain interactions on the ground state are discussed. Results are used for the interpretation of the observed stripe phases in doped antiferromagnets.
\end{abstract}

PACS numbers: 64.60.-i, 71.27.+a, 74.72.-h, 75.10.-b 
It is well known [1] that at low enough temperatures quasi-one-dimensional conductors may undergo a Peierls- or spin-Peierls transition and develop accordingly either a long-range charge- or spin-order. In the case of a discrete lattice model the commensuration effects become important [2]. Exact solutions of related Hartree-Fock problems led to the picture of a solitonic lattice appearing away from half-filling of the bare electron band [3,4]. The nature of a soliton was determined then by a corresponding order parameter which was either lattice deformation, or density of electronic spin. Nevertheless, recently discovered stripe phases in doped antiferromagnets (cuprates and nickelates) [5] have attracted attention to the problem of coupled spin and charge order pararmeters in the electron systems. Numerical mean-field calculations [6,7] suggest a universality of the spin-charge multi-mode coupling phenomenon in repulsive electronic systems of different dimensionalities. On the other hand, those calculations are bound to use small clusters which often makes them inconclusive.

In this paper an exact analytical solution of the Hartree-Fock problem for a onedimensional electron system at and away from half-filling is presented. This solution provides a unique possibility to investigate analytically the structure of the periodic spin-charge solitonic superlattice. It also demonstrates fundamental importance of the higher order commensurability effects, which result in special stability points along the axis of concentrations of the doped holes. Though there is no long-range order in the purely one-dimensional system due to destructive influence of fluctuations, real cuprates are three-dimensional, and therefore, the long-range order survives in the ground state. Hence, we believe, that one-dimensional mean-field solutions contain universal features of the stripe phase, which are stabilized in higher dimensions. We use derived here single-chain analytical solutions as building blocks for the stripe phase in quasi two(three)-dimensional system of parallel chains. In this way we have found that short-range (nearest neighbour) repulsion between doped holes, in combination with effects of magnetic misfit energy between the chains, naturally leads to formation of either "half-filled" or "fully filled" domain walls in the low- or high doping limits respectively. In both cases these walls separate neighbouring antiphased antiferromagnetic domains. The "bi-stripe" solution shows up as well.

The Hubbard Hamiltonian with the hopping integral $t$ and on-site repulsion $U(>0)$ may be written in the form:

$$
H=t \sum_{\langle i, j\rangle \sigma} c_{i, \sigma}^{\dagger} c_{j, \sigma}+U \sum_{i}\left(\frac{1}{4} \hat{n}_{i}^{2}-\left(\hat{S}_{i}^{z}\right)^{2}\right)
$$

Here an identity: $\hat{n}_{i \uparrow} \hat{n}_{i \downarrow}=\frac{1}{4} \hat{n}_{i}^{2}-\left(\hat{S}_{i}^{z}\right)^{2}$ is used. Operators $\hat{n} \equiv \hat{n}_{\uparrow}+\hat{n}_{\downarrow}$ and $\hat{S}^{z}$ are fermionic density and spin (z-component) operators respectively, and $\sigma$ is spin index. Hamiltonian (1) has convenient form for Hartree-Fock decoupling in the presence of the two order parameters, i.e. electron spin- and charge-densities , $m(x)$ and $\rho(x)$ respectively. Single-particle eigenstates and eigenvalues of the Hamiltonian Eq.(1) in the Hartree-Fock approximation can be determined from the Bogoliubov-de Gennes equations derived in [7]:

$$
\hat{H}\left(\begin{array}{l}
\Psi_{\sigma+}(x) \\
\Psi_{\sigma-}(x)
\end{array}\right)=\left(\begin{array}{cc}
-i \frac{d}{d x}+\alpha \rho(x) & -\alpha m(x) \\
-\alpha m(x)^{*} & i \frac{d}{d x}+\alpha \rho(x)
\end{array}\right)\left(\begin{array}{c}
\Psi_{\sigma+}(x) \\
\Psi_{\sigma-}(x)
\end{array}\right)=E /(2 t)\left(\begin{array}{l}
\Psi_{\sigma+}(x) \\
\Psi_{\sigma-}(x)
\end{array}\right),
$$

where $\alpha=U / 4 t$, the Plank constant is taken as unity, and length is measured in the units of the lattice (chain) period $a$. In these units momentum and wavevector are dimensionless, 
and velocity and energy posses one and the same dimensionality. The wave function is defined in terms of the right- left-movers $\Psi_{\sigma \pm}$ as

$$
\Psi_{\sigma}(x)=\Psi_{\sigma+}(x) \exp (i \pi x / 2)+\sigma \Psi_{\sigma-}(x) \exp (-i \pi x / 2)
$$

where $\sigma=\uparrow, \downarrow$, and the Fermi-momentum is $p_{F}=\pi \rho / 2$. In the case of half-filling the average number of electrons per site equals $\rho=1$, i.e. $p_{F}=\pi / 2$. The averages $m(x)$ and $\rho(x)$ are defined as:

$$
\langle\hat{n}(x)\rangle=\rho(x), \quad\left\langle\hat{S}^{z}(x)\right\rangle=m(x) \exp (i \pi x)+m^{*}(x) \exp (-i \pi x) .
$$

In the case of half-filling: $\left\langle\hat{S}^{z}(i)\right\rangle=(-1)^{i} m(i)$, where the order parameter $m(x)$ must be real. The total energy is equal to

$$
\frac{W}{2 t}=\sum_{E<\mu} \epsilon+\int d x \frac{\alpha}{2}\left(m^{*}(x) m(x)-\rho^{2}(x)\right),
$$

where $\epsilon=E / 2 t, \mu$ is a chemical potential. Next, we introduce $\bar{\rho}$ and $\tilde{\rho}$ as

$$
\rho(x)=\bar{\rho}+\tilde{\rho}(x), \quad \int \tilde{\rho}(x) d x=0,
$$

and pass to a new basis $\Psi_{1}, \Psi_{2}$, according to [9], which in fact is the Bloch-wave basis in the periodic charge-density wave potential $U \tilde{\rho}(x)$ (in what follows we drop spin index $\sigma$ ) :

$$
\Psi_{ \pm}(x)=\exp \left(\mp i \alpha \int^{x} \tilde{\rho} d x^{\prime}\right) \Psi_{1,2}(x)
$$

Using this basis we can rewrite Eq.(2) in the form

$$
\hat{H}\left(\begin{array}{c}
\Psi_{1}(x) \\
\Psi_{2}(x)
\end{array}\right)=\left(\begin{array}{cc}
-i \frac{d}{d x} & \Delta(x) \\
\Delta^{*}(x) & i \frac{d}{d x}
\end{array}\right)\left(\begin{array}{l}
\Psi_{1}(x) \\
\Psi_{2}(x)
\end{array}\right)=(E / 2 t-\alpha \bar{\rho})\left(\begin{array}{l}
\Psi_{1}(x) \\
\Psi_{2}(x)
\end{array}\right)
$$

where

$$
\Delta(x)=-\alpha m(x) \exp \left(2 i \alpha \int \tilde{\rho}(x) d x\right)
$$

It is easy to find from the finite band potential theory [8] all formal single-soliton solutions of the eigenvalue problem (7):

$$
\Delta(x)=\epsilon_{0}-i k_{0} \tanh k_{0} x
$$

Here $\epsilon_{0}$ is the energy of the localized level, counted from the chemical potential,

$$
k_{0}=\sqrt{\Delta_{0}^{2}-\epsilon_{0}^{2}}
$$

and $2 \Delta_{0}$ is the gap in the energy spectrum. Notice, that we consider $\Delta_{0}$ and $\epsilon_{0}$ (or equivalent pair of variables) as two independent variational parameters. The reason is that, according 
to Eqs. (2) and (8), there are two independent mean fields "hidden" in $\Delta(x)$. Each of them should obey a self-consistency equation. First, we derive equation for $m(x)$, using Eq. (3):

$$
m(x)+m^{*}(x)=\sum_{E<\mu}\left(\Psi_{+}(x) \Psi_{-}^{*}(x)+c . c .\right)
$$

which can also be rewritten in the $\Psi_{1,2}(x)$ representation Definition given in Eq. (可) leads to a self-consistency equation for the variable part of the charge mean-field $\tilde{\rho}(x) \equiv\langle\hat{n}(x)\rangle-\bar{\rho}$ :

$$
\tilde{\rho}(x)=\sum_{E<\mu}\left(\Psi_{1}^{+}(x) \Psi_{1}(x)+\Psi_{2}^{+}(x) \Psi_{2}(x)\right)-\bar{\rho}
$$

Wave functions of the continuum spectrum are as follows:

$$
\Psi_{1,2}=\frac{ \pm \epsilon \mp \epsilon_{0}+k+i k_{o} \tanh k_{0} x}{2 \sqrt{L} \sqrt{\epsilon\left(\epsilon-\epsilon_{0}\right)-k_{0} / L}} e^{i k x}
$$

the upper (lower) sign on the r.h.s. corresponds to the index "1"( "2"); energy $\epsilon$ and wave vector $k$ are related by equation

$$
\epsilon^{2}=\Delta_{0}^{2}+k^{2}
$$

and $L$ is the length of the system (in units of the lattice spacing).

Simultaneously, wave functions of the localized state with energy $\epsilon_{0}$ become:

$$
\Psi_{1}(x)=\Psi_{2}(x)=\frac{\sqrt{k_{0}}}{2 \cosh k_{0} x} .
$$

For correct summation over the energy levels in all the equations above, periodic boundary conditions are imposed on the wave functions $\Psi_{ \pm}$of the continuum spectrum:

$$
\Psi_{ \pm}(x+L)=\Psi_{ \pm}(x) .
$$

Then, quantization condition follows:

$$
k L+\arctan \frac{k_{0}}{k}=2 \pi n
$$

where $\mathrm{n}$ is an integer. Resulting self-consisting charge and spin components of the singlesoliton state are obtained:

$$
\begin{aligned}
& \tilde{\rho}(x)=\frac{k_{0}}{2 \cosh ^{2} k_{0} x}\left[\nu-1+\frac{2}{\pi} \arcsin \frac{\epsilon_{0}}{\Delta_{0}}\right], \\
& m(x)=-\frac{\epsilon_{0} \pm i k_{0} \tanh k_{0} x}{\alpha} \exp \left[ \pm 2 i \alpha\left(\nu-1+\frac{2}{\pi} \arcsin \frac{\epsilon_{0}}{\Delta_{0}}\right)\left(\frac{\tanh k_{0} x}{2}-\frac{x}{L}\right)\right] \exp (i \chi),
\end{aligned}
$$

where $\nu$ is the filling factor of the localized level $(\nu=0,1,2)$; and $\chi$ is an arbitrary phase. As is evident from Eq. (田), at half filling, i.e. in the commensurate case $\rho=1$, the order parameter $m(x)$ must be real. In order to fulfil this condition in Eq. (17) (to the lowest order in $\alpha$ for the case $\nu=0,2$, and precisely, for $\nu=1$ ) one chooses: $\epsilon_{0}=0$. Eqs. (16), (17) describe structures of the topological spin-charge solitons (kinks), which are either spinless 
with charge $\pm e$ (single electron charge) at $\nu=0,2$, or chargless with spin $1 / 2$ in the case $\nu=1$. In all the cases there are two antiphased antiferromagnetic domains in the system. The $\nu=1$ soliton is the stationary excited state of the undoped system. The same is true in the cases $\nu=0(\nu=2)$, but with one electron removed(added) from(to) the system (i.e. still zero doping in the thermodynamic limit). Important is that when doping with holes or electrons becomes finite, already the ground state of the system possesses periodic "chain" of the alternating spinless solitons and antisolitons of the kind $\nu=0$ or $\nu=2$ (for hole- or electron doping respectively), which are described above. This solitonic superlattice, as we assume, is a one-dimensional analogue of the stripe phase observed in lightly doped cuprates and nickelates [5]. Existence of the superlattice is related to appearance of the central band around $\epsilon=0$, filled with either spinless "holes" $(\nu=0)$ or spinless "electrons" $(\nu=2)$.

In order to find a structure of the ground state at finite doping we calculate the total energy Eq. (5) of the electron system using solutions (16) and (17). We obtain for the potential energy:

$$
\frac{W_{p o t}}{2 t}=\int d x \frac{\alpha}{2}\left(m^{*}(x) m(x)-\rho^{2}(x)\right)=\frac{L \Delta_{0}^{2}}{2 \alpha}-\frac{k_{0}}{\alpha}-\frac{\alpha}{6}\left(\nu-1+\frac{2}{\pi} \arcsin \frac{\epsilon_{0}}{\Delta_{0}}\right)^{2} k_{0}-\frac{\alpha}{2} L \bar{\rho}^{2} .
$$

The other part of the total energy reads:

$$
\frac{W_{e l}}{2 t}=\sum_{E<\mu} \epsilon_{i}=-\frac{L}{\pi}\left(p_{F} \epsilon_{F}+\Delta_{0}^{2} \ln \frac{\epsilon_{F}+p_{F}}{\Delta_{0}}\right)+w,
$$

where $\epsilon_{F}^{2}=p_{F}^{2}+\Delta_{0}^{2}$, and the term $w$ is of the order $L^{0}$.

Minimization of the total energy with respect to $\Delta_{0}$ in the order $\propto L$ gives the usual result [3] :

$$
\Delta_{0}=2 \epsilon_{F} \exp \left(-\frac{1}{\lambda}\right), \quad \lambda=\frac{2 \alpha}{\pi} .
$$

Since our approximation makes sense when $\Delta_{0} \ll \epsilon_{F}$, we conclude that parameter $\alpha=$ $U / 4 t$ should be much less than 1 (weak interaction limit). In this limit we see that part in the potential energy $\int \alpha \tilde{\rho}^{2} / 2$ is of the order $\alpha$ and is much less than other terms, which are of the order $\alpha^{-1}$ or $\alpha^{0}$.

The total energy of the kink state Eq. (5) can be expressed similar to the work [3]:

$$
\frac{W-W_{0}}{2 t}=\Delta_{0}\left[\left(\nu-\frac{2 \theta}{\pi}\right) \cos \theta+\frac{2}{\pi} \sin \theta\right]-\frac{\alpha}{6} \Delta_{0}\left(\nu-\frac{2 \theta}{\pi}\right)^{2} \sin \theta
$$

where $W_{0}$ is the energy of the state without a soliton, $\cos \theta=\epsilon_{0} / \Delta_{0}, \sin \theta=k_{0} / \Delta_{0}$. As we have already seen, $\epsilon_{0}=0$ for the half-filled case, thus leading to $\theta=\pi / 2$.

Minimization of (21) with respect to $\theta$ in the case $\rho \neq 1$ (it is assumed that $|\rho-1| \gg$ $\left.\Delta_{0} / v_{F}\right)$ leads to the equation:

$$
\frac{2}{\pi}\left(\theta-\frac{\pi}{2} \nu\right)\left[\left(1-\frac{2 \alpha}{3 \pi}\right) \sin \theta-\frac{\alpha}{3 \pi}\left(\theta-\frac{\pi}{2} \nu\right) \cos \theta\right]=0 .
$$

Solutions are the same as for the Peierls model and are independent of $\alpha$ when $\alpha \ll 1$. The only nontrivial solution is a solitonic excited state with: 


$$
\nu=1, \quad \epsilon_{0} \approx 0, \quad q \approx 0, \quad W-W_{0}=2 \Delta_{0} / \pi
$$

where $q$ is the charge of a soliton. This chargeless solitonic excitation corresponds to a gradual phase-shift by $\pi / 2$ of the argument of the sine function in the periodic ground state solution given below in Eq. (26). Thus, we see that the structure of the ground state of our model should be similar to the one of the Peierls model [3]. Hence, we can conclude that for the finite hole density $n_{h}=|\rho-1|$ charged $(\nu=0,2)$ solitons form a periodic structure with the spin period $l=2 /|\rho-1|$ equal twice the charge period.

In the lowest order expansion in $\alpha \ll 1$ this structure is described in terms of elliptic functions:

$$
\begin{aligned}
& \tilde{\rho}(x)=\frac{2 K\left(r^{\prime}\right) \Delta_{0} r}{\pi}\left[\operatorname{sn}^{2}\left(\Delta_{0} x / r, r\right)-\frac{1}{r^{2}}\left(1-\frac{E(r)}{K(r)}\right)\right], \\
& m(x)=\Delta_{0} \sqrt{k} \operatorname{sn}\left(\Delta_{0} x / \sqrt{k}, k\right),
\end{aligned}
$$

where $\operatorname{sn}\left(\Delta_{0} x / \sqrt{k}, k\right)$ is the Jakobi elliptic function with the parameter $0<k<1$ defined by $2 K(k) \sqrt{k} / \Delta_{0}=1 /|\rho-1|$, where $K(r)$ and $E(r)$ are complete elliptic integrals of the first and second kind respectively, and $r=2 \sqrt{k} /(k+1), r^{\prime}=\sqrt{1-r^{2}}$. Parameter $k$ varies from $k=1$ at $\rho=1$ where $\operatorname{sn}\left(\Delta_{0} x\right)=\tanh \left(\Delta_{0} x\right)$, to $k \ll 1$ where $\operatorname{sn}\left(\Delta_{0} x / \sqrt{k}, k\right) \sim \sin (\pi|\rho-1| x)$. Simultaneously, $E(0) / K(0)=1$, and $E(1) / K(1)=0$.

In the limiting case of "overdoping": $|\rho-1| \gg \Delta_{0}$, in which case $k \ll 1$ and $K(k) \approx \pi / 2$, one has:

$$
\begin{gathered}
m(x) \approx \frac{\Delta_{0}^{2}}{\pi|\rho-1|} \sin (\pi|\rho-1| x), \\
\tilde{\rho}(x) \approx \frac{\Delta_{0}^{4}}{\pi^{2}|\rho-1|^{3}} \cos (2 \pi|\rho-1| x),
\end{gathered}
$$

in accord with the results of [9] obtained in the limit of main harmonics coupling.

It is known that in any exactly integrable model there is no commensurability effects at the commensurate points [1] :

$$
\left|\rho_{0}-1\right|=m / n
$$

where $m, n$ are relatively prime integers. That is the energy and other system parameters continuously depend on the filling factor $\rho$. But in our case the term $-\alpha \rho^{2} / 2$ in the potential energy (5) violates exact integrability and leads to a pinning of the spin density wave $m(x)$. As a result at any rational point we obtain a decrease in the total energy of the order [11]

$$
\delta w \propto-\alpha e^{-n \text { const }}
$$

To summarise we consider briefly the two-dimensional (2D) case using our 1D results by adding weak interchain interactions. In the lowest order approximation in the interchain hopping integral $t_{\perp}$ the interaction energy is

$$
\delta W=-J \sum_{<i, j>} \int_{0}^{L} d x\left(\cos \left(\varphi_{i}-\varphi_{j}\right)-1\right)+W_{\text {Coulomb }}
$$


where $J \sim t_{\perp}^{2} / \Delta_{0}, \varphi_{i}$ is the phase of a spin-density $m(x)$ on the $i$-th chain, $W_{\text {Coulomb }}$ is the Coulomb interaction energy between the charged kinks (solitons). We suppose for simplicity that the Coulomb interaction decreases rapidly with the distance and take into account only charged kinks on the neighbouring sites of the neighbouring chains, $W_{\text {Coulomb }}=N_{p} q^{2} / \epsilon a^{\prime}$, where $N_{p}$ is the number of pairs of the charged kinks, $\epsilon$ is a dielectric constant, and $a^{\prime}$ is an interchain spacing. In the half-filled system, i.e. in the absence of the charged kinks, the minimum of (30) is achieved when $\varphi_{i}=\varphi_{j}$ for all $i, j$, and we have $\delta W=0$. There are two possible ways to create a periodic solitonic structure (solitonic superlattice) in the doped 2D system. In the first case the charged kinks would reside on every chain, while in the second case only every even (odd) chain would be doped. Compare the energies of these two different configurations.

In the first case we have $\varphi_{i}=\varphi_{j}$ and

$$
\delta W_{1}=W_{\text {Coulomb }}=N_{h} q^{2} / 2 \epsilon a,
$$

where $N_{h}$ is the number of kinks (solitons) and the charge $q$ could be deduced from Eqs. (16) and (24). This is a 2D stripe pattern with filled "domain walls", i.e. having one spinless charged kink per period $a^{\prime}$ perpendicular to the chains direction. In the second case the Coulomb repulsion energy is negligible, but there is an increment in the total energy due to the first term in (30), i.e. due to a magnetic order misfit: $\varphi_{i} \neq \varphi_{j}$. The minimal energy configuration could be achieved now in two ways. One possible pattern corresponds to halffilled "bi-stripe" pattern, seen experimentally in some doped manganites [5]. Namely, the kink - anti-kink pairs of the smallest possible size $\xi$ would form on each even(odd) chain in order to keep the phase-shift of $m(x)$ with respect to the odd(even) chains equal to $2 \pi$. The odd(even) chains remain antiferromagnetically ordered. Then the energy is:

$$
\delta W_{2} \sim N_{h} J \xi
$$

where $\xi$ is of the order of the kink width. $\xi$ monotonically increases from its value $v_{F} / \Delta_{0} \sim$ $e^{1 / \lambda}$ at $\rho=1$, to $v_{F} / \Delta \sim e^{2 / \lambda} \tan \pi|\rho-1| / 2$ in the limit $|\rho-1| \gg e^{-1 / \lambda}[10]$. In the case $\delta W_{1}>\delta W_{2}$ we have that the alternating kink structure is energetically preferable. Notice, that function $\delta W_{1}$ monotonically decreases, while function $\delta W_{2}$ monotonically increases as the function of $|\rho-1|$, therefore the sign in the above inequality may change at some sufficient doping concentration of the holes.

An alternative, half-filled single-stripe pattern may arise when $J \xi \gg 2 \Delta / \pi$, i.e. when $t_{\perp}$ is not too small. In this case the minimum of the energy (30) is achieved at $\varphi_{i}=\varphi_{j}$. For this to be true for any couple of the chains, while $W_{\text {Coulomb }} \approx 0$ being also fulfilled, the doped holes should again reside, say, on every even chain in the form of spinless charged solitons $\nu=0$. But simultaneously, an equal number of the chargeless solitons $\nu=1$ (with spin $\pm 1 / 2)$ must be formed at all the odd chains in order to maintain the condition $\varphi_{i}=\varphi_{j}$. This configuration will be stable if:

$$
W_{s} N_{h}<\frac{q^{2}}{2 \epsilon a} N_{h},
$$

where $W_{s}=2 \Delta / \pi$ is creation energy of the chargeless kink. Notice that since the charge $q$ monotonically decreases from 1 to 0 as function of doping $N_{h}=L|1-\rho|$ (see Eqs. (16),(27)), 
therefore the inequality (33) will be not satisfied at high doping densities, and half-filled to filled stripe transition would be expected in qualitative accord with experiment [5].

Instructive discussions with Jan Zaanen and S.A. Brazovski are highly appreciated by the authors. 


\section{REFERENCES}

[1] G. Grüner, Density Waves in Solids (Addison-Wesley, Reading, MA, 1994).

[2] I.E. Dzyaloshinskii and A.I. Larkin, Sov. Phys. JETP 34, 422 (1972).

[3] S.A.Brazovskii, JETP 78, 677 (1980); S. A.Brazovskii, S. A. Gordyunin, N. N. Kirova, JETP Lett. 31, 457 (1980).q

[4] W.P. Su, J.R. Schrieffer, and A.J. Heeger, Phys. Rev. Lett. 42, 1698 (1979); K. Machida and M. Fujita, Phys. Rev. B 30, 5284 (1984).

[5] S-W. Cheong et al., Phys. Rev. Lett. 67, 1791 (1991); C.H. Chen, S-W. Cheong, and A.S. Cooper, ibid. 71, 2461 (1993); J.M. Tranquada et al., ibid. 78, 338 (1997); J.M. Tranquada et al., Nature (London) 375, 561 (1995).

[6] J. Zaanen and O. Gunnarson, Phys. Rev. B 40, 7391 (1989); V.J. Emery, S.A. Kivelson, and H -Q. Lin, Phys. Rev. Lett. 64, 475 (1990); C. Castellani, C. Di Castro and M. Grilli, ibid. 75, 4650 (1995).

[7] H.J. Schulz, J. Phys. (Paris) 50, 2833 (1989); Phys. Rev. Lett. 64, 1445 (1990).

[8] S.A.Brazovskii, S.I.Matveenko, Sov. Phys. JETP 60, 804 (1984).

[9] S.I. Mukhin, preprint cond-mat/9811409.

[10] S.I.Matveenko, Sov. Phys. JETP 60, 1026 (1984).

[11] I.E.Dzyaloshinskii, I.M.Krichever, JETP 56, 908 (1982). 